\title{
Percepción de universitarios y bachilleres respecto del aprovechamiento tras 17 meses de confinamiento por covid-19
}

\author{
Irene Zapata Silva \\ irene.zs@slp.tecnm.mx \\ María Guadalupe Hernández Sierra \\ maria.hs@slp.tecnm.mx \\ Blanca Estela Ramírez Gámez \\ blanca.rg@slp.tecnm.mx \\ Norma Orocio Castro \\ norma.oc@slp.tecnm.mx \\ María Laura Granja García \\ maria.gg@slp.tecnm.mx
}

\section{Estudiante: Luis Martín Rodríguez Vega \\ L17180359@slp.tecnm.mx}

Tecnológico Nacional de México campus Instituto Tecnológico de San Luis Potosí Soledad de Graciano Sánchez,

S.L.P., México

\section{RESUMEN}

El objetivo del trabajo fue conocer el sentir de los alumnos universitarios y bachilleres luego de 17 meses de confinamiento por COVID-19, con respecto de la experiencia y aprovechamiento de las clases en línea. Es un estudio cuantitativo, se empleó como instrumento de recolección de datos una encuesta en línea a 648 discentes y se utilizó estadística descriptiva para el análisis de la información. Los resultados muestran las ventajas y desventajas percibidas, así como su nivel de aprovechamiento académico dónde, el 54 por ciento manifiesta que disminuyó. Con respecto de los distractores 323 alumnos atienden el celular, 324 pierden interés por la mala calidad de la red. 464 consideran una ventaja el ahorro en el gasto de transporte y alimento, 462 ahorraron en tiempo de traslado y 392 dicen haber descubierto nuevas habilidades en el uso de las TIC. 
El 77 por ciento manifiesta que aumentó la carga de trabajo; sorprendentemente, el 78 por ciento no ha dejado de asistir a las clases virtuales. Luego del análisis de los resultados se percibe la necesidad de que el docente implemente estrategias efectivas para captar, mantener la atención e interactuar con los estudiantes en el momento presente de la clase virtual.

Palabras clave: aprendizaje significativo; aprovechamiento académico; clases virtuales; conectividad; covid-19. 


\title{
Perception of university students and high school graduates after 17 months of confinement by covid-19
}

\begin{abstract}
The objective of the work was to know the feelings of university students and high school students after 17 months confinement by COVID-19, focus to experience online classes. It is a quantitative study, an online survey of 648 students was used as a data collection instrument and descriptive statistics were used for the analysis. The results show the perceived advantages and disadvantages, as well as their level of academic achievement, 54 percent of students mention that it decreased. The analysis showed interesting data about physical distractors, 323 students answer the cell phone, 324 lose interest due to the poor quality of the network. 464 consider saving in transportation and food expenses an advantage, 462 saved in travel time and 392 say they have discovered new skills in the use of information technology. 77 percent mention that the workload increased; surprisingly, 78 percent have not stopped attending virtual classes. After analyzing the results, the need for the teacher to implement effective strategies to capture, maintain attention and interact with students in the present moment of the virtual class is perceived.
\end{abstract}

Keywords: meaningful learning; academic achievement; virtual classes; connectivity; covid-19.

Artículo recibido: 30 noviembre. 2021 Aceptado para publicación: 29 diciembre 2021

Correspondencia: irene.zs@slp.tecnm.mx Conflictos de Interés: Ninguna que declarar 


\section{INTRODUCCIÓN}

A partir del 19 de marzo del 2020 y hasta la fecha, en México se vive una situación de contingencia por la pandemia mundial del COVID-19 por lo que, la instrucción educativa se ha realizado de manera virtual de acuerdo con la indicación publicada en el Diario Oficial de la Federación (Gobernación, 2020), el 16 de marzo del mismo año, dónde el secretario de la Secretaría de Educación Pública (SEP), Esteban Moctezuma Barragán, dio a conocer la suspensión de clases de todos los niveles del Sistema Educativo Nacional. Sin embargo, en el acuerdo presentado no se dieron recomendaciones puntuales, ni un soporte técnico o metodológico por parte de la SEP para llevar a cabo la instrucción (Navarrete, 2020).

El periodo inicial abarcaba a partir del lunes 23 de marzo al viernes 17 de abril, para reanudar labores a partir del lunes 20 de mismo mes, siempre y cuando las condiciones fueran apropiadas para volver a las aulas. Se decidió enviar tareas a los alumnos y evaluar las actividades al regreso, considerando que éste sería en la fecha prevista. Sin embargo, al mes de septiembre de 2021 el retorno no se ha dado en todas las instituciones educativas, ya que la SEP ha planteado que éste se realizará hasta que haya semáforo verde y un alto porcentaje de la población ya vacunada en cada uno los estados.

El gobierno de México se pronunció a favor del regreso a clases presenciales a partir del mes de agosto de 2021 de manera no obligatoria y escalonada.

La suspensión de las clases presenciales cambió de modo abrupto el epicentro alrededor del cual estaba organizado el sistema educativo, teniendo que desplazarse desde las escuelas hasta los hogares, lo que ha representado un desafío inédito para todos los involucrados. Como argumenta (Delgado, P., 2020) "Los tomó a todos por sorpresa, poniendo en evidencia que muchos docentes no tienen las habilidades tecnológicas y la formación necesaria para enseñar en línea". Se encontraron frente al reto de transformar sus contenidos educativos a textos, vídeos y actividades en formato digital. Fueron enviados en muchos casos, a la "guerra sin fusil". Todos están tratando de adaptarse y aprendiendo en este proceso.

Algunas instituciones educativas aprovecharon las plataformas que ya tenían funcionando y muchas otras emprendieron una carrera acelerada para diseñar nuevos portales educativos con contenidos propios. Esta nueva modalidad implicó una desigualdad en el acceso a la educación, los hogares tanto de los docentes como de los alumnos tuvieron 
que adquirir servicio de Internet y computadoras para la realización de su trabajo. Sin embargo, la mayoría de los jóvenes utiliza los teléfonos móviles, que son los dispositivos tecnológicos de mayor penetración.

La aplicación más empleada es el WhatsApp, según se aprecia en el siguiente gráfico:

"Las redes sociales utilizadas para comunicarse con sus compañeros son: el 99\% por WhatsApp, seguida por Facebook y en menor medida por Instagram y Twitter.” (Ríos, 2020).

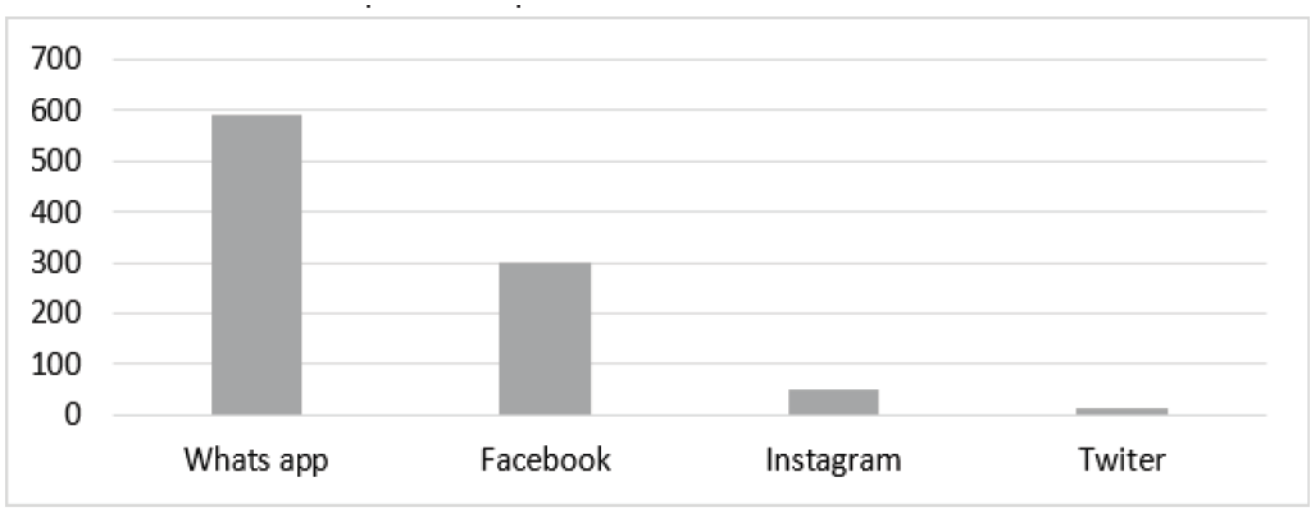

Ante esta realidad fue necesaria la capacitación del cuerpo académico, para lo cual se utilizaron los siguientes medios:

a) Webinars

b) Ciclos de conferencias

c) Foros

d) Tutoriales

e) Cursos abiertos

f) Cursos virtuales de mayor nivel de estructuración

g) Diplomados

h) Certificaciones

\section{ESTRATEGIAS METODOLÓGICAS O MATERIALES Y MÉTODOS}

Para la identificación de los métodos de investigación según refiere (Hernández-Sampieri R., 2018); el estudio realizado es de tipo cuantitativo. Se aplicó una encuesta en línea para conocer el aprovechamiento de las clases en línea durante los 17 meses de confinamiento $\mathrm{y}$ sin el regreso presencial a las instituciones educativas de nivel medio superior $\mathrm{y}$ superior. Para la recolección de los datos y posterior análisis de los mismos, se utilizó la estadística descriptiva. 


\section{Método y organización del trabajo de campo.}

\section{Se llevaron a cabo las siguientes acciones:}

a) Se utilizó Microsoft Forms de Google para la elaboración del cuestionario. La encuesta se diseñó con tres tipos de respuestas:

- De varias opciones

- De solo una opción

- Escala de Likert

b) Para obtener las respuestas de la encuesta, la distribución del enlace se realizó por medios digitales. Se envió a 15 instituciones educativas de Licenciatura y a 7 de Bachillerato, localizadas en doce estados de la República Mexicana. Se obtuvieron 648 respuestas, de las cuales 462 corresponden a estudiantes de nivel superior y 186 de nivel medio superior.

c) Recolección y tratamiento de los datos.

\section{RESULTADOS Y DISCUSIÓN}

En las respuestas obtenidas se muestra que, 186 alumnos, los cuales corresponden al 29 por ciento estudian en nivel medio superior y 71 por ciento (462) del nivel superior.

Figura 1. Nivel de estudios

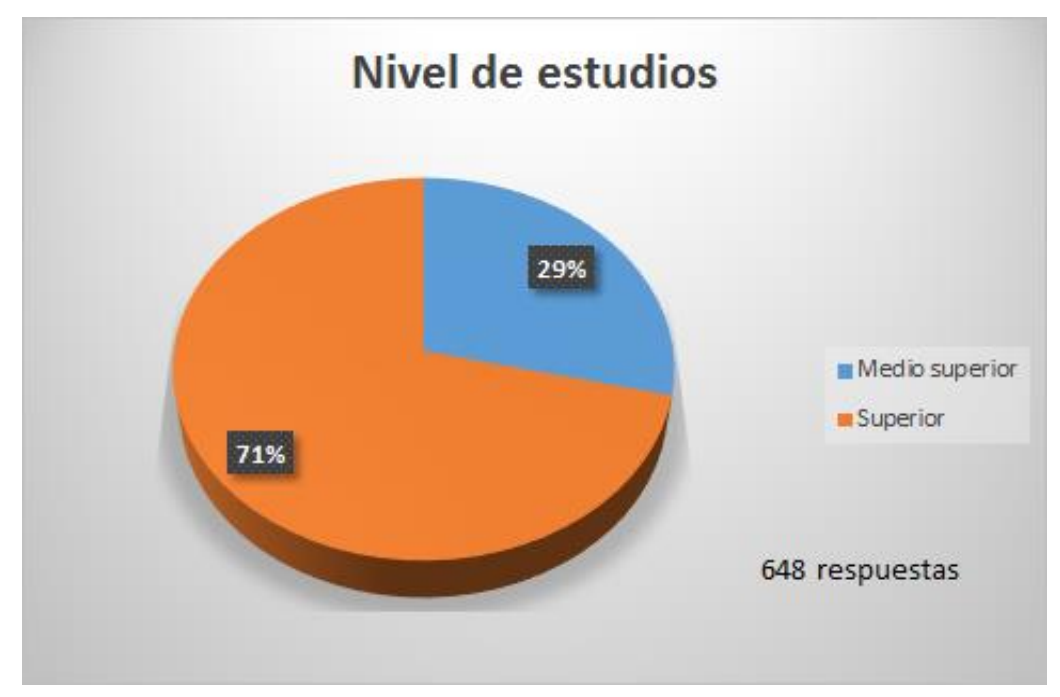

Fuente: Elaboración propia

Desde la percepción de los jóvenes, el aprovechamiento escolar en las clases en línea ha disminuido además de que en el periodo pre-pandemia estaba demeritado. Como lo advierte (Fernández, 2020):

La pandemia provocada por la covid-19 está golpeando a nuestro frágil sistema educativo y los resultados serán desoladores. Los servicios de media 
superior serán afectados, pues antes de la contingencia ya experimentaba su propia crisis: 1) rezagos en aprendizajes prioritarios; 2) problemas de retención escolar; 3) problemas de equidad en ingreso y permanencia; 4) caída histórica de la matrícula de $1.8 \%$; y 5) deficiencia presupuestaria. Para estos servicios, la pandemia "no cayó como anillo al dedo".

En la tabla número 1 y figura 2, se muestran las respuestas de los encuestados a la pregunta ¿Cómo cambió tu nivel de aprovechamiento con la educación en línea?, dónde $87(13 \%)$ respondieron que aumentó, 350 que son el 54 por ciento asegura que disminuyó y $211(33 \%)$ manifiestan que no hubo cambios.

Tabla 1 Cambio en el nivel de aprovechamiento.

\begin{tabular}{|l|c|}
\hline ¿Cómo cambió tu nivel de aprovechamiento con la educación en línea? \\
\hline Aumentó & 87 \\
\hline Disminuyó & 350 \\
\hline No hubo cambio & 211 \\
\hline Total & $\mathbf{6 4 8}$ \\
\hline
\end{tabular}

Fuente: Elaboración propia

Figura 2. Nivel de aprovechamiento

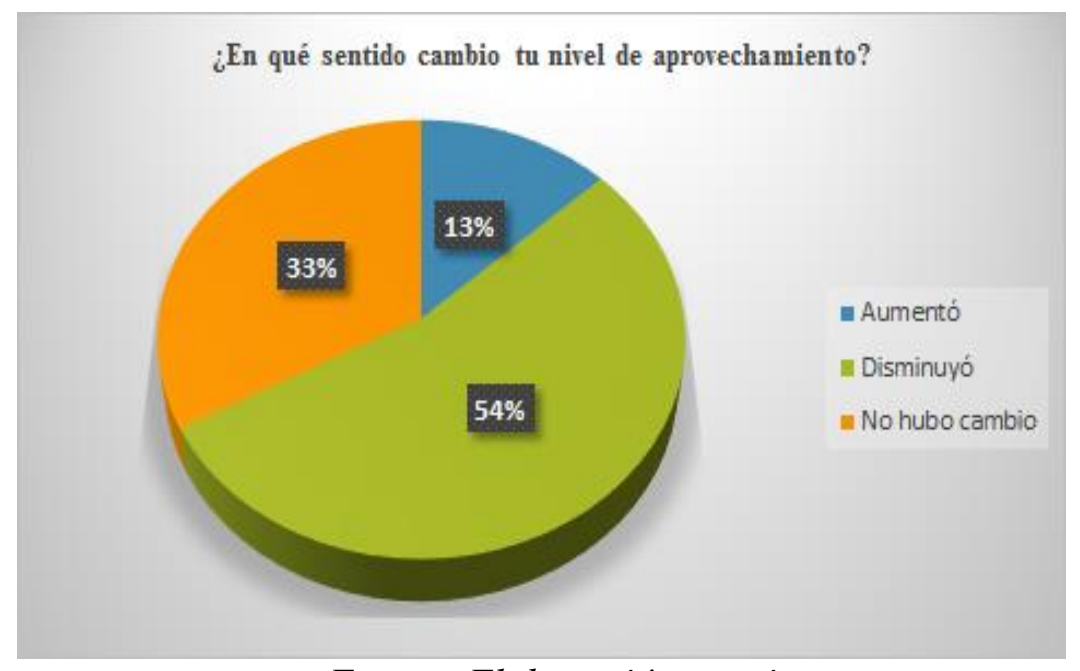

Fuente: Elaboración propia

Es de suponerse que los alumnos en clases virtuales se distraen con facilidad por estar atendiendo múltiples tareas, resultando un bajo nivel de comprensión y por consiguiente un pobre desempeño académico. En el artículo publicado por (Lepp, 2019) se señala: "En los cursos presenciales, un maestro físicamente presente y la presencia de estudiantes 
concienzudos ayudan a hacer cumplir las políticas del aula y las normas de comportamiento contra la multitarea".

A diferencia de un salón de clases, tomar la lección en la comodidad del hogar, en el transporte y hasta en la calle, motiva que los estudiantes se distraigan con facilidad y pierdan el interés en el tema, ya que algunas veces se limitan solo a escuchar la explicación. Además, factores externos como la mala o nula señal de Internet y el que se tenga que compartir el dispositivo con los hermanos ocasiona inseguridad para externar dudas e inasistencias. Otro factor que mencionan los estudiantes tiene referencia con la estandarización en el uso de plataformas educativas e-Learning. Indican que deben atender varias modalidades de interacción con sus profesores.

Los estudiantes se apropian del conocimiento de manera diferente; tienen preferencias y modos individuales en cómo procesan y perciben la información, a esto se le conoce como estilo de aprendizaje. Lo anterior se refiere a cómo aprenden y no a la cantidad de conocimiento adquirido y, en muchas ocasiones, cómo les resulta más fácil y agradable este proceso.

De acuerdo con (García-Allen, 2021), "Los estilos de aprendizaje son una mezcla de factores cognitivos, afectivos y fisiológicos característicos" de cada persona, que sirven como indicadores relativamente estables de cómo el alumno percibe, interactúa y responde a la apropiación del conocimiento.

En el estudio realizado el estilo predominante corresponde al visual con un 65 por ciento, seguido del auditivo con un 35 por ciento y solamente el 5 por ciento de la población mundial es kinestésico. Razón por la cual 271 de los encuestados refieren necesitar ver la explicación del profesor para comprender los temas. Figura 3.

Figura 3. Factores que motivan cambios en el aprovechamiento académico

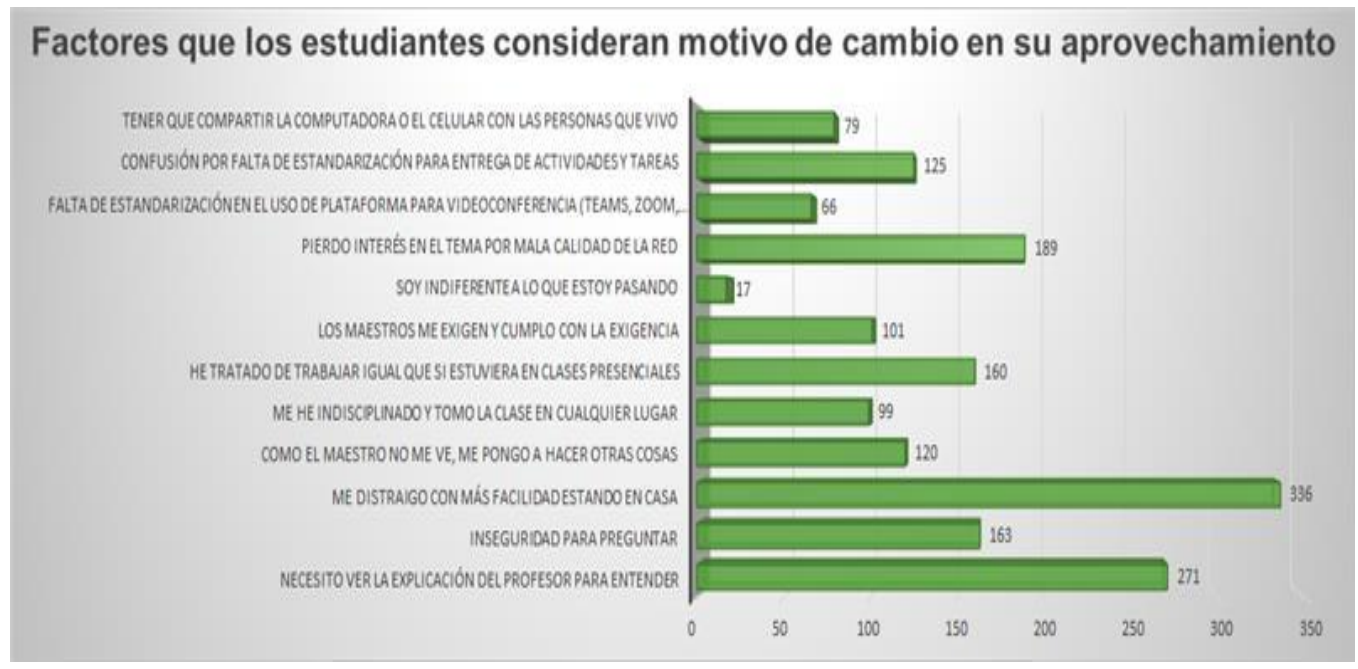

Fuente: Elaboración propia 
Como comenta (Morin, A., 2021), muchas familias están lidiando con preocupaciones financieras. La situación económica que éstas presentan se ha visto mermada por lo tanto no pueden proporcionar a sus hijos los recursos necesarios para tener conexión de Internet o un dispositivo electrónico que los conecte a sus clases en línea. Algunos estudiantes tienen necesidad de trabajar y así ayudar económicamente a su familia y tal vez por su horario de trabajo tengan que ausentarse de clase.

De acuerdo con (Ayala, 2020), El porvenir académico de los estudiantes no es alentador, las dificultades económicas abren una brecha digital que traerá consecuencias en su desarrollo y bienestar.

Como se observa en la figura 4, las situaciones económicas por las que los estudiantes faltan a clases alcanzan un 5 por ciento. El 10 por ciento por la necesidad de trabajar y el 7 por ciento no asiste por falta de recursos tecnológicos (Internet, computadora).

Figura 4. Razones por las que faltan los estudiantes a clases virtuales

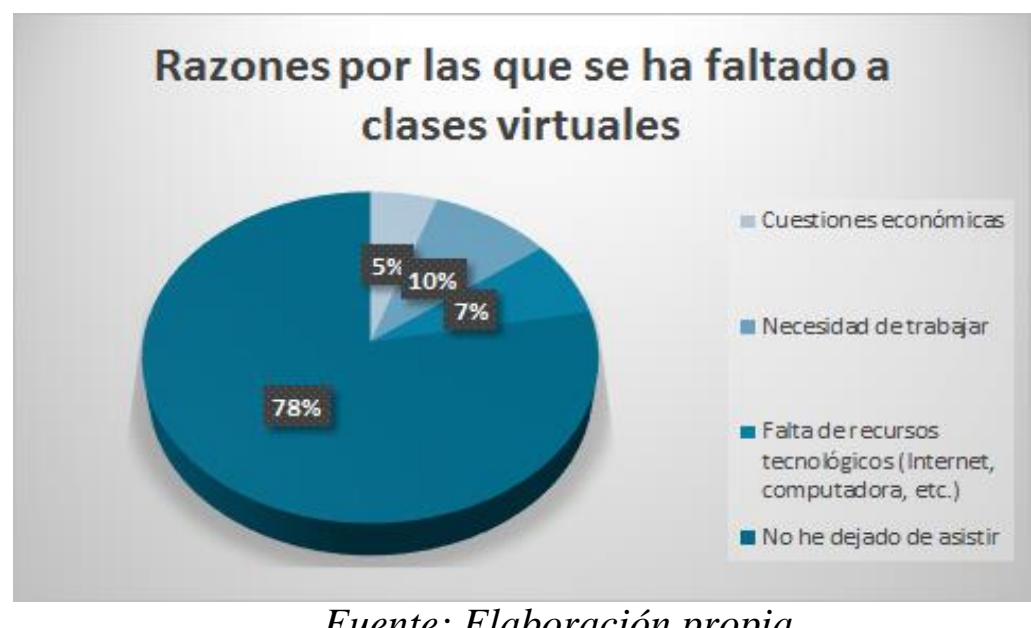

Al principio con la suspensión de las clases presenciales por pandemia, los estudiantes pensaron que estarían de vacaciones utilizando el Internet para diversión: plataformas streaming, redes sociales, juegos en línea. En cuanto los profesores y las instituciones educativas organizaron sus actividades académicas de manera virtual, asignaron a los alumnos tareas y los requirieron para conectarse en horarios específicos a sesiones para la enseñanza del programa de estudios.

Como lo mencionan (Flores, G., Torre, L., 2020) en su publicación titulada Universitarios ahogados en tareas online: "Las clases remotas los han asfixiado, lo que pensaban serían unas largas vacaciones se tornaron en sobrecarga de trabajos. “

Así lo refieren los estudiantes, ya que el 77 por ciento de ellos manifiesta un aumento en 
la carga de trabajo académico y el 23 por ciento considera que se siente igual que en las clases presenciales, como se señala en la figura 5.

Figura 5. Aumento de la carga de trabajo en clases virtuales

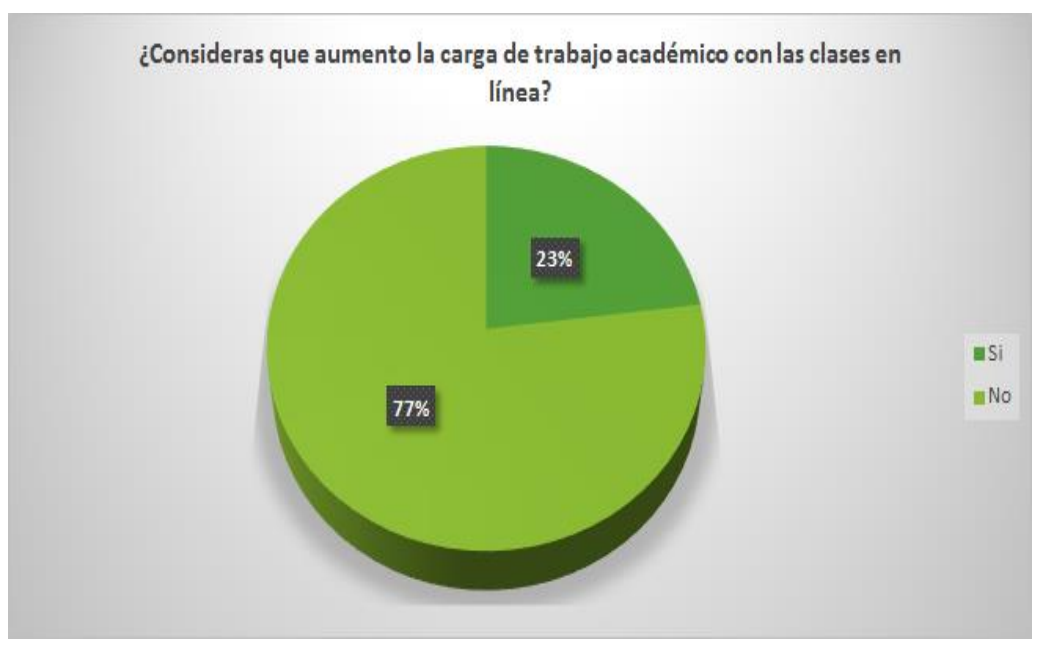

Fuente: Elaboración propia

En el cuestionamiento sobre las ventajas de las clases en línea, la que obtuvo mayoría de respuestas con 464 la referente al ahorro de gastos de transporte y alimentación, seguida de 462 en ahorro de tiempo de traslado. La Universidad del Valle de Puebla (Puebla, 2021) menciona en su análisis sobre las ventajas de esta modalidad: "Ya no necesitas preocuparte por cruzar la ciudad, con una mochila cargada de libros, para llegar a tu salón, desde que tomaron mayor importancia las clases en línea, puedes estudiar desde la sala de tu casa o en el comedor...."

Otro factor es la adquisición de nuevas habilidades informáticas, con 392 opiniones; han aprendido a utilizar hojas de cálculo, presentadores de diapositivas, procesador de texto, realizar vídeos, buscar información, y otras herramientas tecnológicas de manejo de hardware y software. Los empleadores solicitan la capacidad de gestionar autónomamente un equipo informático. Figura 6.

Figura 6. Ventajas de las clases virtuales

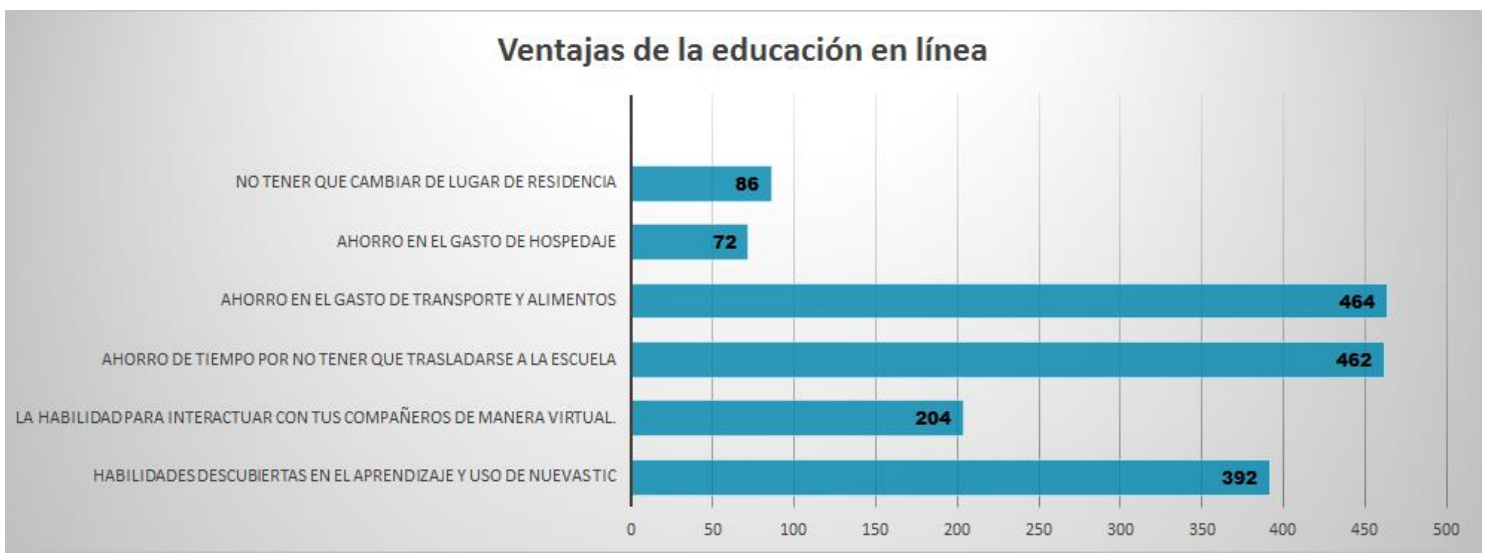


En las desventajas los encuestados señalaron como primer punto, con 324 opiniones, la mala calidad de la comunicación en la red y con 323 la distracción personal de la clase en línea, la cual se da en diferentes formas como lo es que contesten correos electrónicos, envíen mensajes de texto, vean vídeos, jueguen vídeo juegos, entablen conversaciones en redes sociales, escuchen música, naveguen en la red. Está respuesta demostró la conducta multitarea que los estudiantes están adquiriendo, lo que se ha convertido en un impedimento para lograr un aprovechamiento eficiente (Guijosa, C., 2019).

306 coincidieron en menor socialización con los compañeros y maestros, pues en las clases online se tiene un contacto más limitado. Figura 7.

Figura 7. Desventajas de las clases virtuales

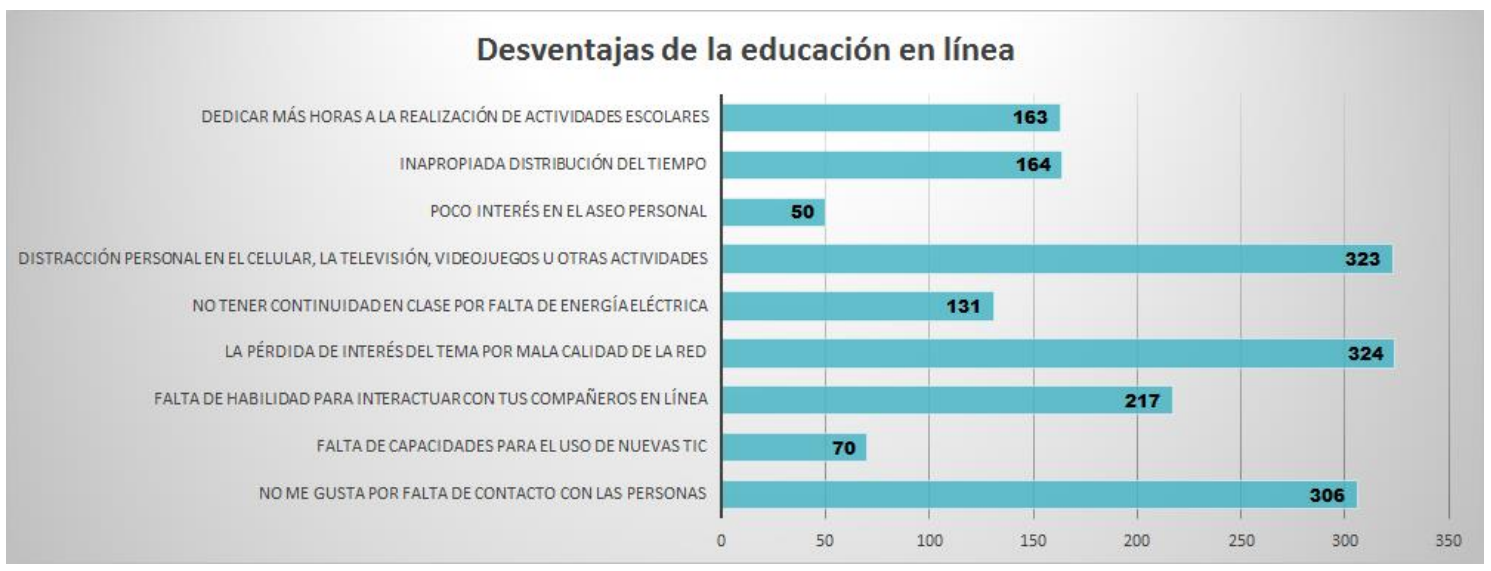

Fuente: Elaboración propia

\section{CONCLUSIÓN O CONSIDERACIONES FINALES}

El retorno presencial al aula se ha dado parcialmente, no como era antes de la pandemia y tal vez nunca vuelva a ser así. De ahí la importancia de que los maestros sigan presentes y conectados tanto como sea posible, utilizando reforzamiento positivo con sus alumnos, procurando con ello que los estudiantes no se sientan aislados en esta nueva normalidad, ya que actualmente aún no es posible reunirse en el mismo espacio.

Es necesario fomentar en ellos mismos y en sus alumnos la resiliencia, con el objetivo de recuperarse de las adversidades e intentar producir aprendizajes significativos. Como lo indica (Chavarría, 2020) esta modalidad, "Permite a los alumnos desarrollar habilidades que en el método presencial les tomaría más tiempo, algunas de ellas son la resiliencia, atención visual y auditiva, trabajar de manera autónoma, estructuración de ideas, entre otros."

Las estimaciones del (GRUPO BANCO MUNDIAL. Educación, 2020) para 2021, en el 
sentido del aumento de abandono escolar y la falta de acceso para algunos alumnos, no son alentadoras. A medida que las escuelas reabran estos indicadores podrán disminuir. Incluso mientras contribuyen con la lucha contra la pandemia, los sistemas educativos deben lanzar una campaña contra la pérdida de los aprendizajes. Sin una acción efectiva es probable que estás pérdidas sean graves, tanto por la desvinculación de los estudiantes y deserción escolar como porque la mayoría de los hogares no estarán en capacidad de apoyar los aprendizajes como lo hacen las escuelas. Es necesario que la enseñanza y el aprendizaje continúen (Liberman, 2020). Si bien la educación en línea no es la solución perfecta, se puede ver que es necesario que instituciones, docentes, alumnos y familia estén dispuestos a adaptarse a esta nueva forma de educar y a tomar aspectos útiles de la misma (Mendoza, 2020).

La pandemia ha traído además de situaciones difíciles, nuevo conocimiento a estudiantes, docentes e inclusive padres de familia, volviéndose diestros en el manejo de la tecnología digital. Actualmente con el confinamiento, algunas empresas han implementado actividades en teletrabajo y han tenido que capacitar a su personal en estas TIC. En esta modalidad educativa es seguro que algunos de los jóvenes universitarios adquirieron habilidades en el manejo y uso de estas herramientas, por lo que no tendrán inconvenientes en este sentido para adaptarse a esta modalidad. Como lo cita (Cívico, 2021), "En las últimas décadas, la tecnología se ha integrado en nuestras vidas de manera gradual y vinculándose, cada vez más, a nuestra relación con el entorno en diferentes esferas".

A los discentes de bachillerato y universitarios encuestados, les agrada la comodidad que da estudiar desde casa porque tienen horarios más flexibles y pueden atender necesidades de su casa mientras estudian, además del ahorro de tiempo y gasto por no tener que trasladarse a la institución. Aunque la falta de contacto con sus compañeros es imprescindible para su desarrollo integral, por lo tanto según los resultados del estudio de (MEXTUDIA, 2020) "Los estudiantes continuarán sus estudios, pero no quieren continuar en modalidad a distancia".

Encontrándose los estudiantes en casa, los cambios en el patrón de consumo de Internet doméstico han aumentado, ocasionando que las redes se saturen y dejen de operar de manera eficiente.

Una de las problemáticas de la educación en línea es la deficiente conectividad. Según 
publica (Zavala, M., 2020):

"En plena pandemia por COVID-19, el mal servicio de Internet y telefonía móvil motivó que se duplicarán las quejas ante el Instituto Federal de Telecomunicaciones (IFT). De acuerdo con el informe del tercer trimestre del 2020, un total de 8 mil 715 inconformidades llegaron al Instituto por medio del sistema soy usuario, de las cuales 30 por ciento corresponden al servicio de Internet, 27 por ciento a servicios de telefonía fija más Internet fijo, y 18.5 por ciento a servicios de telefonía móvil”.

Luego del análisis de los resultados se percibe la necesidad de que el docente implemente estrategias efectivas para captar, mantener la atención e interactuar con los estudiantes en el momento presente de la clase virtual, asegurando que se han apropiado del conocimiento y que el aprendizaje está siendo significativo; toda vez que no los tiene frente a él, mitigando así las desventajas que presenta este nuevo modelo de formación académica. Sin embargo, se sigue ensayando, readaptando, creciendo y avanzando, quedando un largo camino por recorrer. La construcción del conocimiento continúa preparando y rediseñando nuevas formas de educar.

\section{REFERENCIAS BIBLIOGRAFICAS}

Ayala, S. \&. (2020). Herramientas digitales y reconversión de actividades en los hogares mexicanos. Vicisitudes de la escuela en casa en tiempos de COVID 19. En G. N. Palos, Efectos sociales, económicos, emocionales y de la salud ocasionados por la pandemia del COVID-19: Impactos en Instituciones de Educación Superior y en el Proceso de Enseñanza Aprendizaje (Vol. 1, págs. 83-112). San Luis Potosí, San Luis Potosí, México: Plaza y Valdés Editores. Recuperado el 26 de septiembre de 2021, de

https://www.researchgate.net/profile/Mario-

Venegas/publication/349413322_Estudio_exploratorio_sobre_educacion_remota_d urante_COVID-

19_en_Universidad_Tecnologica/links/604c4258458515e529a40589/Estudioexploratorio-sobre-educacion-remota-durante-COVID-19-en

Chavarría, M. (20 de agosto de 2020). Colegio Castillo de Chapultepec. Recuperado el 22 de septiembre de 2021, de SE PUEDE LOGRAR UN APRENDIZAJE 100\% SIGNIFICATIVO CON LA EDUCACIÓN A DISTANCIA: https://www.ccch.edu.mx/se-puede-lograr-un-aprendizaje-100-significativo-con-la- 
educacion-a-distancia/

Cívico, A. C. (1o. de mayo de 2021). Jóvenes y uso problemático de las tecnologías durante la pandemia: una preocupación familiar. Hachetetepé. Revista científica de educación

doi:https://doi.org/10.25267/Hachetetepe.2021.i22.1204

Delgado, P. (6 de abril de 2020). Observatorio de Innovación Educativa del Tecnológico de Monterrey. (T. d. Monterrey, Editor) Recuperado el 22 de septiembre de 2021, de Padres, alumnos y docentes enfrentan los retos de adaptarse a la educación en línea: https://observatorio.tec.mx/edu-news/educacion-online-retos-escuela-en-casa

Estrada, M. (17 de septiembre de 2021). Universidad del Bosque. Recuperado el 30 de septiembre de 2021, de Impacto de las clases virtuales en los procesos de enseñanza y aprendizaje: https://www.unbosque.edu.co/centro-informacion/noticias/impactode-las-clases-virtuales-en-los-procesos-de-ensenanza-y

Fernández, M. H. (8 de julio de 2020). Nexos. Recuperado el 21 de septiembre de 2021, de Jóvenes con un futuro sombrío: media superior ante la pandemia: https://educacion.nexos.com.mx/jovenes-con-un-futuro-sombrio-media-superiorante-la-pandemia/

Flores, G., Torre, L. (1o. de abril de 2020). Universitarios ahogados en tareas online. El Sol de Tampico. Recuperado el 25 de septiembre de 2021, de https://www.elsoldetampico.com.mx/local/regional/adelantan-pago-de-mas-de-17mil-becas-5046587.html

García-Allen, J. (2021). Psicología y Mente. (B. Regader, Editor) Recuperado el 25 de septiembre de 2021, de Los doce estilos de aprendizaje: ¿en qué se basa cada uno?: https://psicologiaymente.com/desarrollo/estilos-de-aprendizaje

Gobernación, S. d. (Ed.). (16 de marzo de 2020). Diario Oficial de la Federación. (Red de publicaciones oficiales mexicanas) Recuperado el 20 de septiembre de 2021, de https://www.dof.gob.mx/nota_detalle.php?codigo=5589479\&fecha=16/03/2020

GRUPO BANCO MUNDIAL. Educación. (mayo de 2020). Recuperado el 27 de septiembre de 2021, de COVID-19: IMPACTO EN LA EDUCACIÓN Y RESPUESTAS DE POLÍTICA PÚBLICA: https://openknowledge.worldbank.org/bitstream/handle/10986/33696/148198SP.pd $\mathrm{f}$ ? sequence $=6 \&$ is Allowed $=\mathrm{y}$ 
Guijosa, C. (26 de febrero de 2019). Obervatorio de Innovación Educativa del Tecnmológico de Monterrey. (T. d. Monterrey, Editor) Recuperado el 21 de septiembre de 2021, de La distracción, un freno para la educación en línea: https://observatorio.tec.mx/edu-news/la-distraccion-un-freno-en-la-educaciononline

Hernández-Sampieri R., F. P. (2018). Metodología de la Investigación. Las rutas cuantitativas, cualitativa y mixta. (1a. ed.). México: Mc Graw Hill.

Lepp, A. B. (13 de enero de 2019). College Students' Multitasking Behavior in Online Versus Face-to-Face Courses. (K. S. University, Ed.) SAGE OPEN, enero-marzo 2019, 1-9. doi:https://doi.org/10.1177/2158244018824505

Liberman, J. L.-B. (27 de abril de 2020). Education for Global Development. Recuperado el 17 de septiembre de 2021, de ¿Siguen aprendiendo los estudiantes durante el COVID-19? La respuesta podría obtenerse a través de la evaluación formativa: https://blogs.worldbank.org/es/education/siguen-aprendiendo-los-estudiantesdurante-el-covid-19-la-respuesta-podria-obtenerse

LinkiaFP Formación profesional oficial. (9 de marzo de 2021). Recuperado el 23 de septiembre de 2021, de Las 8 grandes ventajas de las clases online: https://linkiafp.es/blog/ventajas-clases-online/

Lovón, M. \&. (30 de septiembre de 2020). Repercusiones de las clases virtuales en los estudiantes universitarios en el contexto de la cuarentena por COVID-19: El caso de la PUCP. Propósitos y Representaciones, $\quad 8$ (SPE3). doi:http://dx.doi.org/10.20511/pyr2020.v8nSPE3.588

Mendoza, L. (2020). Lo que la pandemia nos enseño sobre la educación a distancia. (U. I. México, Ed.) Revista Latinoamericana de Estudios Educativos, 50(ESPECIAL), 343-352. doi:https://doi.org/10.48102/rlee.2020.50.ESPECIAL.119

MEXTUDIA. (24 de julio de 2020). Recuperado el 28 de septiembre de 2021, de ¿Cómo afectó el Confinamiento a estudiantes y profesores?: https://mextudia.com/comoafecto-el-confinamiento-a-estudiantes-y-profesores/

Morin, A. (2021). Child Mind Institute. Recuperado el 25 de septiembre de 2021, de 5 razones por las que los estudiantes no están participando en el aprendizaje a distancia: https://childmind.org/es/articulo/5-razones-por-las-que-los-estudiantes-no-estanparticipando-en-el-aprendizaje-a-distancia/ 
Murcia, M. (20 de enero de 2021). Los papás de Gabriel \& Adrián. Recuperado el 25 de septiembre de 2021, de ¿Cómo identificar los estilos de aprendizaje en niños?: https://gabrielyadrian.com/tipos-estilos-aprendizaje-ninos/

Navarrete, Z. M. (2020). Políticas implementadas por el gobierno mexicano frente al COVID-19. El caso de la educación básica. (U. Iberoamericana, Ed.) Revista Latinoamericana de Estudios Educativos, L(Especial), 143-172. doi:https://doi.org/10.48102/rlee.2020.50.ESPECIAL.100

Puebla, U. d. (2021). Ventajas de estudiar en línea. Recuperado el 24 de septiembre de 2021, de https://blog.uvp.mx/ventajas-de-estudiar-en-linea/

Ríos, V. H. (2020). Dispositivos, herramientas digitales y plataformas más utilizadas por universitarios mexicanos durante el primer trimestre del confinamiento por el COVID-19. En G. N. Palos, Efectos sociales, económicos, emocionales y de la salud ocasionados por la pandemia del COVID-19: Impactos en Instituciones de Educación Superior y en el Proceso de Enseñanza Aprendizaje (Vol. 1, págs. 355 376). San Luis Potosí, San Luis Potosí, México: Plaza y Valdés Editores. Recuperado el 21 de septiembre de 2021, de https://www.researchgate.net/profile/MarioVenegas/publication/349413322_Estudio_exploratorio_sobre_educacion_remota_d urante_COVID-

19_en_Universidad_Tecnologica/links/604c4258458515e529a40589/Estudioexploratorio-sobre-educacion-remota-durante-COVID-19-en

Romero, L., Salinas, V. \& Mortera, F. (abril de 2010). Estilos de aprendizaje basados en el modelo de Kolb en la educación virtual. (G. M. Dávila, Ed.) Apertura, 2(1), 72 85. Recuperado el 24 de septiembre de 2021, de http://www.udgvirtual.udg.mx/apertura/index.php/apertura/article/view/21/30

Zavala, M. (4 de diciembre de 2020). Se disparan quejas por mal servicio de Internet. Incrementaron 100 por ciento los reportes de fallas en pandemia. El Heraldo de México. Recuperado el 29 de septiembre de 2021, de https://heraldodemexico.com.mx/nacional/2020/12/4/se-disparan-quejas-por-malservicio-de-internet-232161.html 